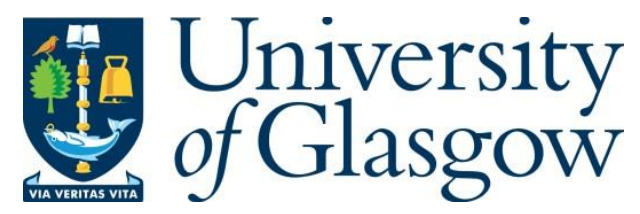

Azqueta-Gavaldon, A. (2017) Financial Investment and Economic Policy Uncertainty in the UK. In: 1st International Conference on Internet of Things and Machine Learning, Liverpool, UK, 17-18 Oct 2017, Article 40, ISBN 9781450352437.

There may be differences between this version and the published version. You are advised to consult the publisher's version if you wish to cite from it.

(C) Association for Computing Machinery 2017. This is the author's version of the work. It is posted here for your personal use. Not for redistribution. The definitive Version of Record was published in the Proceedings of the 1st International Conference on Internet of Things and Machine Learning, Liverpool, UK, 17-18 Oct 2017, Article 40, ISBN 9781450352437, https://doi.org/10.1145/3109761.3158380.

http://eprints.gla.ac.uk/163132/

Deposited on: 09 August 2018

Enlighten - Research publications by members of the University of Glasgow http://eprints.gla.ac.uk 


\title{
Financial Investment and Economic Policy Uncertainty in the UK
}

\author{
Andres Azqueta-Gavaldon \\ Adam Smith Business School, \\ Glasgow University \\ Rm 101, 12 Southpark Terrace, \\ Glasgow G12 8LG \\ a.azqueta-gavaldon.1@research.gla.uk
}

\begin{abstract}
UK based financial firms following Brexit reported net disinvestment of 15 billion pounds. This was the fifth time financial disinvestment occurred since the production of this data: 1987. Parallel to this event, Economic Policy Uncertainty (EPU) in the UK experienced its biggest rise during Brexit June 2016. This note studies the relationship between EPU and its particular components and financial investment. I find that overall EPU and specifically fiscal policy, monetary policy, geopolitical, regulation and liquidity uncertainty have the highest negative sensitivity to financial investment.
\end{abstract}

\section{CCS CONCEPTS}

- Information systems $\rightarrow$ Retrieval task and goals; Evaluation of retrieval results $\bullet$ Applied computing $\rightarrow$ Economics; Forecasting

\section{KEYWORDS}

Unsupervised machine learning, Latent Dirichlet Allocation (LDA), Economic Policy Uncertainty (EPU), Financial Investment, Forecast

\section{INTRODUCTION}

Net disinvestment of 15 billion pounds was reported by financial firms following Brexit (insurance companies, pension funds, and trusts). This was the fifth time disinvestment occurred since the production of this data started in 1987 [1]. Parallel to this event, Economic Policy Uncertainty in the UK which measures the uncertainty regarding which and when economic policies will take place experienced its most prominent peak during Brexit [2].

This note attempts to study the relationship between EPU and financial investment in the UK. To this end, I focus on the impact of individual components of economic policy uncertainty on financial investment. While it has been proven a strong relationship between corporate investment and overall policy uncertainty [2-3], none to the best of my knowledge concentrate on financial investment. In total, financial firms based in the UK control 4 trillion of assets and are engaged in a considerable volume of investment activity to fund their operations [1].
The main contribution of this note is the use of an unsupervised machine-learning algorithm to compute a news-based economic policy uncertainty index for the UK. This methodology [4], allows unveiling individual components of policy uncertainty to be tested against financial investment. An exercise using a VectorAutoregressive approach - commonly used in finance for forecasting - reveals that fiscal and geopolitical uncertainty explain the biggest drop in net financial investment.

\section{POLICY UNCERTAINY IN THE UK}

I measure policy uncertainty for the UK using the approach proposed by [4], which is an alternative to Baker's et al. (2016) news-based policy uncertainty index. This alternative approach uses an algorithm that unveils the themes of articles without the need for prior knowledge about their content. This allows the researcher to classify each article according to a singular category of economic policy uncertainty (e.g. fiscal, monetary or trade) and construct the overall policy uncertainty index simply by adding them. The main advantage with respect to the traditional method is the availability of individual sub-indices to enrich the study. Given the complexity of the current uncertainty episodes lived in the UK, failing to disentangle the specific sources of uncertainty might result in an incomplete analysis.

The unsupervised machine learning algorithm, called Latent Dirichlet Allocation (LDA) [5], is a generative probabilistic model that labels textual documents with a variety of topics. Each topic is described by a list of words with a high probability of belonging to a given topic. Therefore, the model infers the distribution of words that define a topic, while simultaneously annotating articles with a distribution of topics. The model recovers these two distributions by obtaining the model parameters that maximize the probability of each word appearing in each article given the total number of topics K. The probability of word $\mathrm{w}_{\mathrm{i}}$ occurring in an article is:

$P\left(w_{i}\right)=\sum_{j=1}^{K} P\left(w_{i} \mid z_{i}=j\right) P\left(z_{i}=j\right)$

where $\mathrm{z}_{\mathrm{i}}$ is a latent variable indicating the topic from which the $i$ th word was drawn, $P\left(w_{i} \mid z_{i}=j\right)$ is the probability of word $w_{i}$ being drawn from topic $j$, and $P\left(z_{i}=j\right)$ is the probability of drawing a word from topic $\mathrm{j}$ in the current article. Intuitively, $\mathrm{P}(\mathrm{w} \mid \mathrm{z})$ 
indicates which words are important to a topic, whereas $\mathrm{P}(\mathrm{z})$ states which of those topics are important to an article. The goal is therefore to maximize $P\left(w_{i} \mid z_{i}=j\right)$ and $P\left(z_{i}=j\right)$ from equation (1). Nonetheless, a direct maximization is susceptible of local maxima and slow convergence [6]. Following [4], I use online variational Bayes as proposed by [7]. This method approximates the posterior distribution of $P\left(w_{i} \mid z_{i}=j\right)$ and $P\left(z_{i}=j\right)$ using an alternative and simpler distribution: $\mathrm{P}(\mathrm{z} \mid \mathrm{w})$, and associated parameters. $^{1}$

I run this algorithm in all news articles describing overall economic uncertainty (those containing any form of the terms economy and uncertainty) from the following newspapers: The Financial Times and The Times. The retrieval tool used was Nexis, an online database of news articles. The total number of news articles associated with any form of these two terms from January 1997 to June 2017 was 49,175.

Consistent with previous studies [4], I filter the textual data by removing stopwords, convert all words into lower cases, and transform each word into its root (stemming). Finally, to find the most likely value of topics $\mathrm{K}$ for this specific corpus, I use the likelihood method. This method consists of estimating the likelihood of the probability of words for a different number of topics $\mathrm{P}(\mathrm{w} \mid \mathrm{K})$ empirically. This probability cannot be directly estimated since it requires summing over all possible assignments of words to topics but can be approximated using the harmonic mean of a set of values of $P(w \mid z, K)$, when $z$ is sampled from the posterior distribution [6]. I compute the likelihood estimation for 20, 30, 40, 50 and 60 topics. Table 1 shows the results of these estimations. As can be seen, the optimal number of topics is $\mathrm{K}=$ 30. After $K=30$, the log likelihood score drops steadily, which assures us that we have found the global maximum.

Table 1: Number of topics and log-likelihood scores. Units in $10^{6}$

\begin{tabular}{l|ccccc}
\hline & 20 & 30 & $\mathbf{4 0}$ & $\mathbf{5 0}$ & $\mathbf{6 0}$ \\
\hline $\log P(w / K)$ & -22.8 & -20.2 & -22.3 & -25.5 & -27.1 \\
\hline
\end{tabular}

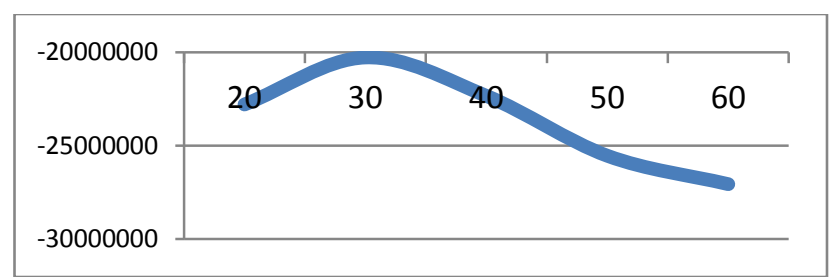

Figure1: Visual representation of the number of topics and log-likelihood scores from Table 1.

\footnotetext{
${ }^{1}$ For more details about the implementation, see [8].
}
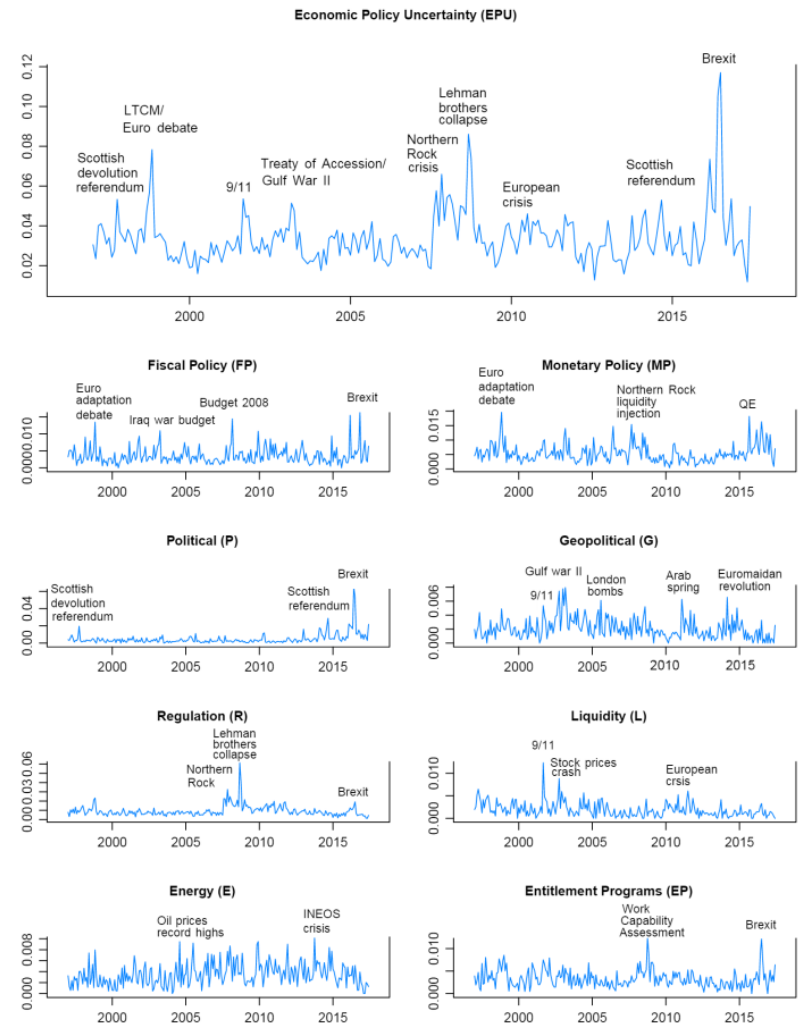

Figure 2: Policy Uncertainty index and its categories build using The Financial Times and The Times from Jan 1997 until June 2017.

Figure 2 shows the evolution of the overall policy uncertainty index and each of its eight sub-indices from Jan 1997 to June 2017 (included). To produce each time series I follow the approach of [4]: Firstly, each article was labelled according to its most representative topic (the topic with the highest percentage in the article). Secondly, a raw count of the number of articles for every topic in each month was produced. Since the number of articles is not constant over time, I divided each raw time-series by the total number of articles containing the word today each month. The EPU index is then the sum of the monthly normalized timeseries of the topics that are assigned to each EPU category.

Overall policy uncertainty (top graph in Figure 2) exhibits clear spikes around events known to increase policy-related uncertainty, such as recessions, geopolitical events (e.g. Gulf War II, London bombings and the Arab spring) or episodes of high political uncertainty (e.g. Scottish referendum for independence and Brexit). Besides, the eight individual components offer in detail which category is behind each shock. For example, fiscal policy and monetary policy uncertainty are responsible for the spike in 
overall EPU at the end of 1998, when Britain was discussing whether or not to join the Euro. Moreover, these three categories also account to a big extent of the rise in uncertainty surrounding Brexit (June 2016). Additionally, geopolitical uncertainty is behind the advance in overall policy uncertainty at the start of the Gulf War II (April 2003), whereas liquidity uncertainty is responsible for the spike around 9/11 which produced a shock in financial markets' liquidity worldwide [9].

\section{FINANCIAL INVESTMENT AND UNCERTAINTY}

To investigate the relationship between EPU and financial investment, I estimate a Vector Auto-regression (VAR). VAR analysis is useful when characterizing dynamic links between time series, as it detects co-movements among them and accounts for possible feedback loops between them [3].

I follow the procedure of [2] and specify a VAR using the policy uncertainty indices (normalized by their sample standard deviation), the natural log of the FTSE index, the Bank of England interest rate, employment rate and net financial investment. Including the stock market index mitigates concerns of endogeneity because stock markets are forward-looking and stock prices react to many sources of information [2]. The data for all economic indicators come from the ONS, the Bank of England and the London Stock Exchange.

The VAR is run quarterly from 1998 Q1 to 2017 Q2 using the number of lags suggested by the Bayesian Information Criterion (BIC). I reject serially correlated residuals and test for stability across the system to ensure that Impulse Response Functions (IRF) can be calculated. 2 To calculate each IRF, I obtain orthogonal shocks using the Cholesky decomposition based on the exact variable ordering previously described. Because each uncertainty index is normalized by its standard deviation, each IRF can be interpreted as the behavior through time of financial investment when each uncertainty type increases (experiences a positive shock) by one standard deviation.

\footnotetext{
${ }^{2}$ Note that since we are interested in examine the relationships between variables, I do not take the first differences to guarantee stationarity among variables. Since doing so would throw information on any long-run relationship between the series away [10]. Stability of the system guarantees that the impulse response functions converge to the steady state. None the less, results do not change significantly when first differences of each variable is computed.
}
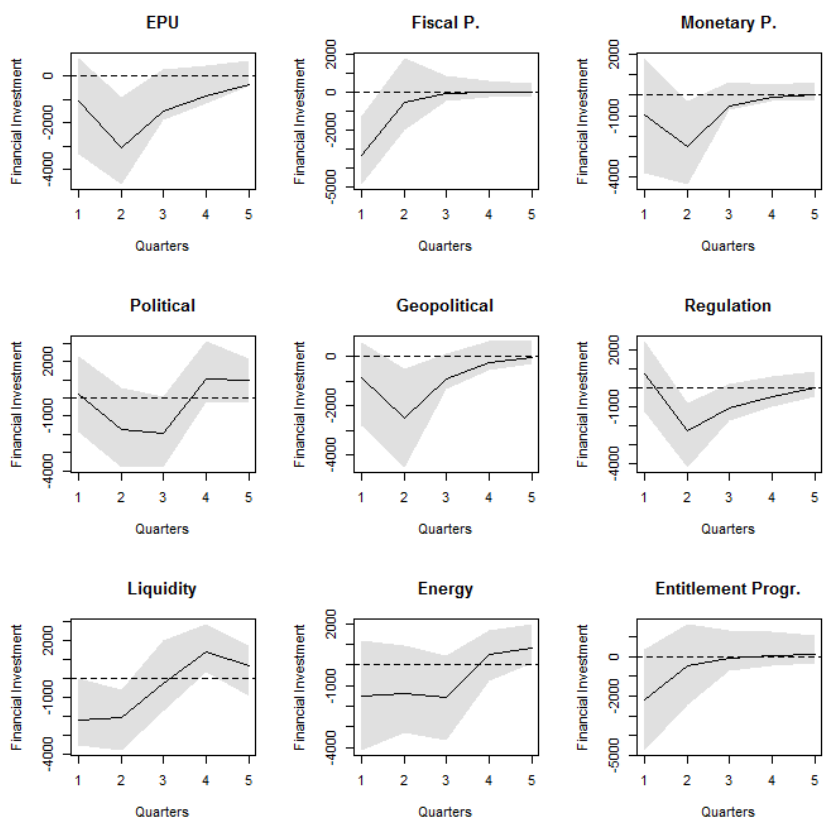

Figure 3: Impulse Response Functions for net financial investment to an EPU and uncertainty sub-indices unit standard deviation shock. Grey bands indicate $90 \%$ confident intervals based on 1,000 bootstrap replications.

Figure 3 show some interesting results. There is an adverse and significant effect of aggregate EPU on financial investment. At its peak, the drop in investment accounts $£ 2,500$ for every standard deviation rise in overall EPU. This is equivalent to $1 / 5$ of the financial investment standard deviation during the sample. Moreover, we observe that the negative effect of policy uncertainty on financial investment extends to at least five quarters into the future.

Regarding individual uncertainty sub-indices, the impact on net financial investment is dissimilar: while fiscal policy, monetary policy, geopolitical, regulation and liquidity uncertainty seem to condition financial investment significantly, energy and entitlement programs do not. The adverse effect is particularly strong in the case of fiscal and geopolitical uncertainty. In regards to political uncertainty, we observe a continuous drop in net investment for three quarters, although this drop is at the margin of being significant.

Interestingly, we observe a rebound on financial investment in many IRF: after a steady decline in financial investment as a result of a positive policy uncertainty shock, financial investment bounces back beyond the steady state to later decline towards it. The rebound dynamic is in line with the theory, which suggests that once uncertainty vanishes, firms undertake the pending investment [2]. This behavior is particularly notable after a shock in political and liquidity uncertainty. 


\section{CONCLUSIONS}

The literature on policy uncertainty has mainly considered the effects of the aggregate implications of this one on investment. In this note, I take a different angle by focusing on the individual categories of economic policy uncertainty - measured through an unsupervised machine learning algorithm - in determining net financial investment. Results, based on quarterly Vector Autoregressive models over the period Q1 1998- Q2 2017 indicate that there is a negative link between financial investment and uncertainty embedded in economic narratives regarding policy changes. Fiscal and geopolitical uncertainties are the sub-indices that show the highest negative co-movements with financial investment. They are closely followed by regulation, liquidity, monetary policy and political uncertainty. These results are robust to the inclusion of the stock market index which captures forwardlooking, confidence and business cycles information.

\section{ACKNOWLEDGMENTS}

I thank Charles Nolan, Campbell Leith, Spyridon Lazarakis, Simon Naitram, Jorge Gonzalez Paule, Diego Azqueta as well as participants at the International Conference on Internet of Things and Machine Learning (IML 2017) in Liverpool, for valuable comments.

\section{REFERENCES}

[1] ONS, 2017. MQ5: Investment by insurance Companies, Pension Funds and Trusts.

[2] Baker, S., Bloom, N., and David, S. 2016. Measuring Economic Policy Uncertainty. The Quarterly Journal of Economics, 131(4).
[3] Gulen, H. and Ion, M. 2016. Policy Uncertainty and Corporate Investment. The Review of Financial Studies. 29(3).

[4] Azqueta-Gavaldon A. 2017. Developing news-based economic policy uncertainty index with unsupervised machine learning. Economics Letters 158: 47-50.

[5] Blei, D. M., Ng, A., and Jordan, M. I. 2003. Latent Dirichlet Allocation. Journal of Machine learning Research 3, 993-1022

[6] Griffiths, T., Steyvers, M. 2004. Finding scientific topics. Proceedings of the National Academy of Sciences of the United States of America (PNAS). 101 (Supplement 1): 5228-5235. April 2004.

[7] Hoffman, M. D., Blei, D. M., and Bach, F. 2010. On-line learning for latent Dirichlet allocation. Neural Information Processing System.

[8] Řehůřek, R., and Sojka, P. 2010. Software Framework for Topic Modelling with Large Corpora. Proc. LREC 2010 Workshop on New Challenges for NLP Frameworks

[9] Posner E., and Vermeule A. 2009. Crisis Governance in the Administrative State: 9/11 and the Financial Meltdown of 2008. The University of Chicago Law Review, 76(4): 1613-1682.

[10] Brooks C. 2014. Introductory Econometrics for finance. Second edition, Cambridge University Press: New-York 